\title{
Metabolic changes in donor corneas during cold storage- challenging stereotypes
}

\author{
Tomasz Kryczka ${ }^{1,2 *}$, Jacek P. Szaflik ${ }^{3}$, Jerzy Szaflik ${ }^{3,4}$ and Anna Midelfart ${ }^{2,5}$ \\ ${ }^{1}$ Department of Medical Biology, Medical University of Warsaw, Warsaw, Poland \\ ${ }^{2}$ Department of Neuroscience, Faculty of Medicine, Norwegian University of Science and Technology, Trondheim, Norway \\ ${ }^{3}$ Department of Ophthalmology, Medical University of Warsaw, Warsaw, Poland \\ ${ }^{4}$ Warsaw Eye Bank, Warsaw, Poland \\ ${ }^{5}$ Department of Ophthalmology, University Hospital, Trondheim, Norway
}

\begin{abstract}
It has been recently shown that cold storage at $2-8^{\circ} \mathrm{C}$ is not neutral towards metabolic properties of the cornea. The aims of this study were to reveal: the kinetics of biochemical changes in human corneas during cold storage and the time of storage when the preserved corneas show the biggest biochemical similarities to in vivo ones. Twenty human corneas were obtained post-mortem from ten 60 to 71 -year-old donors and preserved in Eusol- $\mathrm{C}$ at $+4^{\circ} \mathrm{C}$ for 14 days. The control samples were the tissues stored at $-80^{\circ} \mathrm{C}$ immediately after collecting them from cadaver eye globes. The daily changes in metabolic profiles of the samples were investigated with HR MAS 1H NMR spectroscopy. 25 metabolites were detected and assigned in the corneas. The significant differences in metabolic profiles between Eusol$\mathrm{C}$-preserved and non-preserved tissues, and between the corneas stored for 14 days comparing to 7 -days samples, were revealed. The main differences between the samples were related to the levels of ATP, formate, lactate, glycine and butyrate in the tissues. The corneas preserved for 2 weeks were shown to have the biochemical contents more resembling the in vivo ones than the corneas preserved for one week. The results of this study suggest that from the biochemical point of view the cornea transplantation or any other procedure associated with a removal of the cornea from the storage medium should be performed after two weeks of the hypothermic preservation in Eusol-C.
\end{abstract}

\section{Introduction}

A recent report on human corneas preserved in a commercially available medium at $2-8^{\circ} \mathrm{C}$ has revealed that the hypothermic storage for 8 days decreased the differences between the biochemical contents of the tissues collected at different post-mortem times [1]. However, the next study showed that the above was possible only under the condition that the donors were not affected by any chronic disease. The samples from patients with idiopathic liver cirrhosis or cardiovascular disease differed biochemically from corneas obtained from healthy donors, both before and after the Eusol-C storage [2]. It was quite a surprise as these specific diseases that were not directly related to the function of the eye, were shown markedly affecting corneal biochemistry. Moreover, some of the biochemical abnormalities assigned in the corneas before storage were reduced or nullified after 8 days of the preservation. Interestingly, the between-donors biochemical differences were not accompanied by differences in endothelial cell densities in the investigated corneas [1,2].

The main assumption of ophthalmologists is to provide a transplant procedure with the cornea graft of the best quality to ensure the success of the procedure. According to the European Eye Bank Association or its analogous American association [3,4], the donor cornea must fulfill a number of criteria where the most important are the negative serological test results and the cellular formation of epiand endothelium with the proper cellular density of the latter one. The biochemical status of the cornea was never considered to be important in a standard graft evaluation $[3,4]$. However, the reports by Kryczka et al. $[1,2,5]$ impair the thesis about the conservative properties or functions of the hypothermic storage systems. Then, the question appears how the biochemical content of the cornea changes from day to day during the cold storage? To answer this question the human corneas from healthy donors were preserved in the Eusol-C based hypothermic storage system and everyday collected samples were investigated with HR MAS (High Resolution Magic Angle Spinning) ${ }^{1} \mathrm{H}$ NMR (Proton Nuclear Magnetic Resonance) spectroscopy.

\section{Material and methods}

The study adhered to the tenets of the Declaration of Helsinki and local regulations with regard to the human studies and the use of human tissues and organs, and has been filed with the Bioethics Committee of the Medical University of Warsaw which has acknowledged the protocol of the study and has raised no objection.

\section{Corneas}

Twenty human corneas were obtained from ten 60-71 years old donors 4-12 hours post-mortem. No systemic or chronic disease, or recent medication was reported in donors. None of the donors was a

Correspondence to: Tomasz Kryczka, MSc, MD, PhD, Department of Medical Biology, Medical University of Warsaw Ul. Nowogrodzka 73, 02 018 Warszawa, Poland, Tel: +48 604315103; Fax: +48 226253223; E-mail: kryczka.tomasz@gmail.com

Key words: cornea grafting, cornea biochemistry, cold storage, metabolomics, NMR spectroscopy

Received: June 02, 2015; Accepted: June 12, 2015; Published: June 15, 2015 
heavy alcohol drinker, or was infected with a hepatitis virus, or suffered from an autoimmune disease or cancer. After evaluation by slit lamp biomicroscopy of the cornea quality compliance with European Eye Bank Association criteria [3], corneal buttons of $10 \mathrm{~mm}$ diameter (calliper-measured) were excised free of sclera and limbal rim. Then, $1 / 8^{\text {th }}$ of cornea (pizza slice) was separated and immediately frozen at $-80^{\circ} \mathrm{C}$ until analyzed. These samples were assigned as the control corneas. The remaining tissue was placed in the Eusol-C-based cold storage system at $+4^{\circ} \mathrm{C}$. Paired corneal tissues obtained from the same donor were kept in the storage media for two different storage times: 7 - and 14 days. In the 7 -days storage group every day $1 / 8^{\text {th }}$ of the cornea was separated from the remaining tissue, rinsed with cold $\left(+4^{\circ} \mathrm{C}\right)$ sterile $0.9 \%(\mathrm{w} / \mathrm{v}) \mathrm{NaCl}$ solution and frozen at $-80^{\circ} \mathrm{C}$. In the second storage group the same procedure of freezing the sample was applied on the 14-th day, as described above. The corneas used for this study were disqualified for transplantation due to minor epithelial damage inflicted upon dissecting.

\section{High-Resolution Magic Angle Spinning proton NMR (HR MAS ${ }^{1} \mathrm{H}$ NMR) spectroscopy}

HR MAS ${ }^{1} \mathrm{H}$ NMR spectroscopy was run on a 14.1 T model Avance DRX600 Bruker spectrometer (Bruker BioSpin GmbH, Rheinstetten, Germany) operating at $600.132 \mathrm{MHz}$. Just prior to the analysis, the frozen samples were cut into pieces and immersed in cold $\left(+4^{\circ} \mathrm{C}\right) 25$ $\mathrm{mM}$ solution of sodium $\left[2,2,3,3-\mathrm{d}_{4}\right] 3^{\prime}$-trimethylsilylpropionate in $\mathrm{D}_{2} \mathrm{O}$ in a $4 \mathrm{~mm}$ diameter $(50 \mu \mathrm{l}$ capacity) zirconia rotor. The spectra were recorded at $+4^{\circ} \mathrm{C}$ using a $4 \mathrm{~mm}{ }^{1} \mathrm{H} /{ }^{13} \mathrm{C}$ MAS probe. The samples were spun at $5000 \mathrm{~Hz}$ and the number of scans was 512 . Water suppression was done employing a pre-saturation selective pulse. Exponential line broadening of $0.3 \mathrm{~Hz}$ was used. Carr-Purcell-Meiboom-Gill spectra analysis was performed using MestreNova v. 5.1.0 software (Mestrelab Research, Santiago de Compostela, Spain). Peak areas were measured using absolute integrals and were normalised by the wet weight of the measured samples (weight range of corneal samples immediately frozen: 7.3-12.3 mg; stored for: 1 day: 7.0-12.2 mg, 2 days: 7.5-12.4 mg, 3 days: $7.2-12.1 \mathrm{mg}, 4$ days: 7.2-12.4 mg, 5 days: 7.5-12.3 mg, 6 days: 7.1$12.2 \mathrm{mg}, 7$ days: $7.4-12.3 \mathrm{mg}, 14$ days: $7.6-12.5 \mathrm{mg}$ ) and assignments of the metabolites in the spectra were performed as described earlier $[1,6]$.

\section{Statistical analysis}

Paired corneal tissues obtained from the same donor and immediately frozen at $-80^{\circ} \mathrm{C}$ before placing in the storage media were considered two control groups. The significance of differences between individual means of both control groups was tested by an independent Student's t-test. As no statistical differences were revealed (data not shown) the control samples obtained from the group of corneas stored for 7 days were assumed the control for further analyses.

${ }^{1} \mathrm{H}$ NMR spectral data were first transferred into Unscrambler software (CAMO, Oslo, Norway) for principal component analysis (PCA). The spectral region 0.3-10.0 ppm of samples collected for 0-7 days and 14 days served as the data input. The number of principal components for cross-validation analysis was assessed by the leverage correction validation method as the number of components that gave the minimal total validation $\mathrm{X}$-variance. Next, the cross-validation method with mean centering was employed. To reveal grouping and the relationship between samples, the score plot of the first principal component (PC1) vs. the second principal component (PC2) was interpreted.

Detection limits for the studied metabolites are relatively high (in $\mu \mathrm{M}$ range) in HR MAS ${ }^{1} \mathrm{H}$ NMR spectroscopy. The absence of any of these metabolites was unlikely, and using metabolite titers of " 0 " for those below the respective detection limit would distort the outcome of statistical analysis. Hence, such 'null' titers were omitted.

Metabolite content data were analyzed by repeated measures oneway ANOVA with time of cornea storage in medium as the repeated measure factor. Then the Mauchly test was done for sphericity assessment. If sphericity was not met the Greenhouse-Geisser correction was applied. Then pairwise comparisons for all metabolites were done with Bonferroni correction.

\section{Results}

Twenty-five low molecular weight metabolites were assigned in the studied HR MAS ${ }^{1} \mathrm{H}$ NMR spectra; for a representative spectrum see Figure 1.

\section{PCA results}

Principal components 1 (PC1) and 2 (PC2) accounted for 61\% and $23 \%$, respectively, of the total variation in the NMR spectra of the samples. The score plots of PC1 vs. PC2 showed considerable dispersion along the PC1 axis. High PC1 score was associated with high ATP and formate contents and low glycine contents; the reverse was true for low PC1 score (Figure 2).

Control samples, as well as corneas preserved for 14 days, showed a shift toward higher PC1, while the majority of corneas preserved in cold storage medium for one week were associated with low PC1 values. High PC2 score was related to relatively high lactate and butyrate contents. The shift toward low PC1 values combined with the shift toward low PC2 values yielded noticeably poorer distinction grouping of corneas preserved for 7 days in Eusol-C. In the score plots of NMR spectra of the corneas that were stored in medium no further grouping patterns of the samples were observed (Figure 2).

\section{Amino acids}

Statistical analysis revealed significantly higher levels of alanine (day 1 of storage) and glutamine (1-2-days samples) in comparison to the control, while the reverse was true for methionine (2-7-days samples). The samples kept for 14 days in the storage media revealed lower levels of alanine, glutamine and glutamate than the control

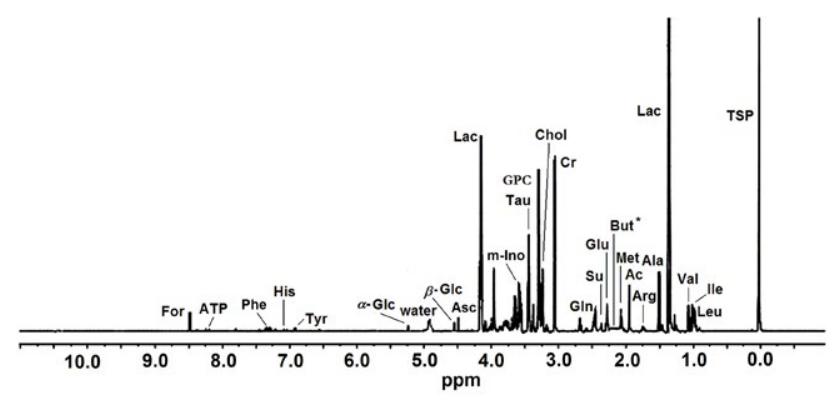

Figure 1. A representative HR MAS 1H NMR spectrum of intact human cornea sample. The ppm values were assigned using sodium [2,2,3,3-d4]3'-trimethylsilylpropionate (TSP) as the reference substance at $0 \mathrm{ppm}$. Ac, acetate; Ala, alanine; $A r g$, arginine; $A s c$, ascorbate; $A T P$, adenosine triphosphate; But, butyrate; Chol, choline; $C r$, creatine; For, formate; b- Glc b-glucose; a-Glc, a-glucose; Glu, glutamate; Gln, glutamine; GPC, glycerophosphocholine; His, histidine; Ile, isoleucine; Lac, lactate; Leu, leucine; Met, methionine; m-Ino, myoinositol; Phe, phenylalanine; $S u$, succinate; Tau, taurine; Tyr , tyrosine; Val, valine. *Presumed location of But that was not detected in the control group corneas. 

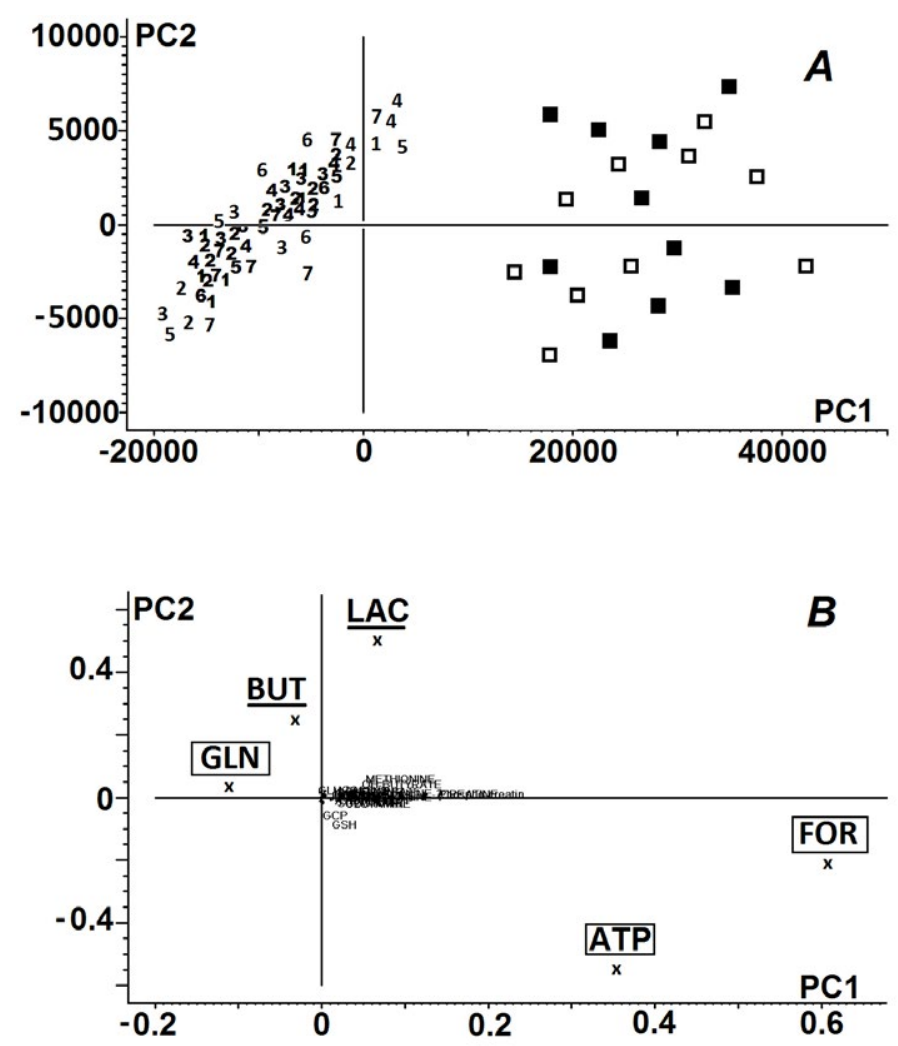

Figure 2. Principal component analysis of donor corneas. Panel A: Score plot of the 1st $v s$. the 2 nd principal component of ${ }^{1} \mathrm{H}$ NMR spectra of human corneas; numeric symbols '1-7' indicate the days of storage of the tissues within the first week, $(\square)$ - the corneas preserved in the storage medium for 14 days, $(\square)$ control corneas. Panel B: Loading profile of the principal components of the ${ }^{1} \mathrm{H}$ NMR spectra of the corneas. Metabolites described in lower cases in the loading profile indicate negligible influence of these compounds on the grouping pattern. The biochemical compounds that served as PC1 were marked with the square frames. The biochemical compounds that served as PC2 were underlined. For metabolite labels see legend for Figure 1.

samples. In addition, alanine and glutamine levels in the samples stored for 14 days were also lower than the respective levels of metabolites in the 7-days samples. Glutamate and phenylalanine were below the detection limit in the samples kept in Eusol-C for 2-7 and 1-7 days, respectively (Figure 3 ).

\section{Non-amino acid low molecular weight metabolites}

Statistical analysis revealed that the levels of ATP, formate in the samples kept in Eusol-C for 2-7 days and glycerophosphocholine (stored for 2 and 6-7 days) were significantly lower than in the control. The samples kept in the cold storage for 14 days revealed significantly higher levels of creatine, formate, myo-inositol and succinate in comparison to the control and the latter three metabolites, as well as butyrate, were also significantly elevated in comparison to the levels of respective compounds in the 7 -days samples. The level of creatine was below the detection threshold in the tissue sample stored for 7 days, as well as in the 2-5-days samples. Ascorbate and butyrate were not detected in the 14-days and control samples, respectively (Figure 4).

The ECD measurements of all corneas involved in the study showed the values between $2350-2950$ cells $/ \mathrm{mm}^{2}$. No significant ECD differences between the groups of samples were revealed.

\section{Discussion}

The recent reports indicate that the high demand for corneal tissue is only partially covered by the network of eye banks. The local laws and customs, political attitude and public awareness make the donor cornea more or less accessible in different states [7-10]. The most of the eye banks are not capable of obtaining locally the sufficient number of donor corneas to cover this demand. To secure the sufficient number of corneal tissues for grafting some eye banks set higher age limits than 75 years [11] or the minimum ECD values of 2,000 cells $/ \mathrm{mm}^{2}$ [12], or buy transplant tissues in other countries. The latter procedure sometimes requires some extension of the storage time to complete the dispatching and adaptation of the corneal cells to a new eye bankrelated environment. Therefore, the research on the feasibility of transferring the donor tissue from cold storage to organ culture to increase the lifespan of corneas has become one of the leading topics in ophthalmology [13,14].

It is a common view that the transfer of corneal tissue from the in vivo (or ex vivo, donor) environment to the artificial storage medium is not too harmful for the cells; the most survive the procedure $[11,15]$. The goal of the storage system is to provide an artificial environment that mimics the natural one and ensures cellular viability and low levels of cellular or molecular damage. However, the storage medium always represents a stressful environment for the donor tissue as no composition of the medium can replace the content of the natural
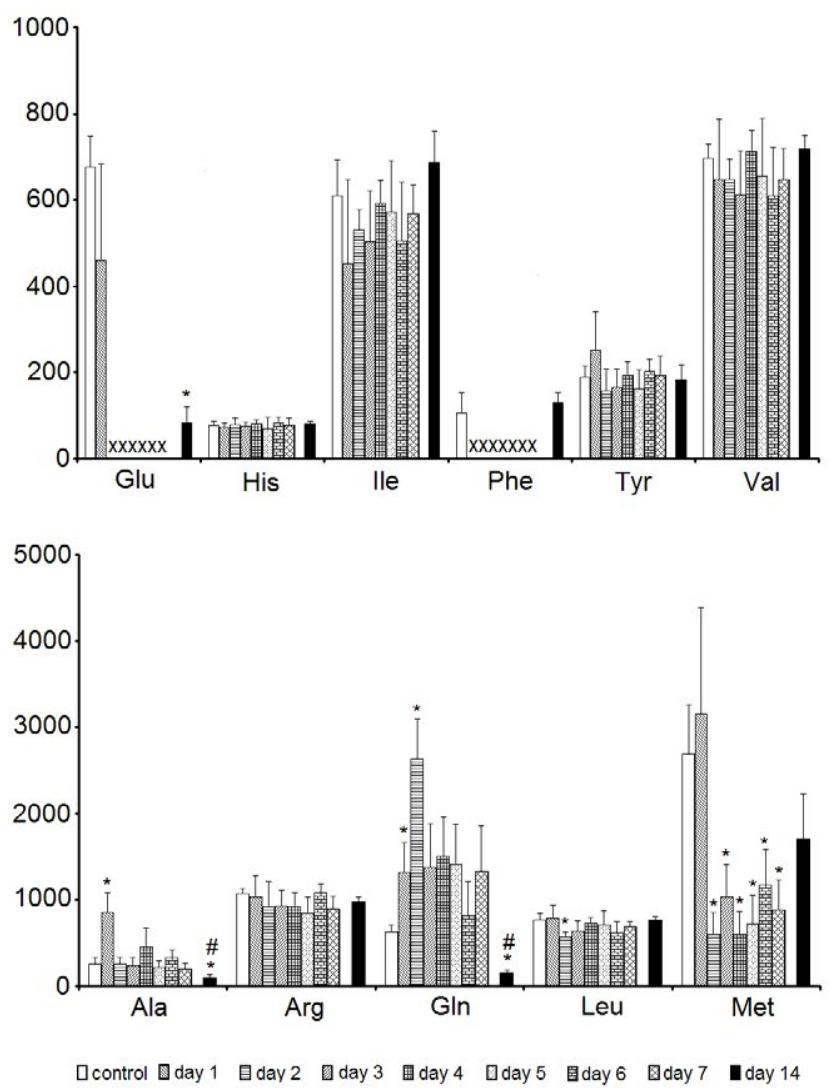

Figure 3. The effect of time of storage on the contents of assigned free amino acids in corneas. Data are means \pm SEM (NMR peak integrals $/$ mg tissue). $*_{-}$p $<0.05 v s$. control; $\#$ - $\mathrm{p}<0.05$, tissue preserved for 14 days $v s$. the ones preserved for 7 days; $\mathrm{X}$ - no detectable metabolite peak in the respective NMR spectra. For amino acid labels see legend for Figure 1. 

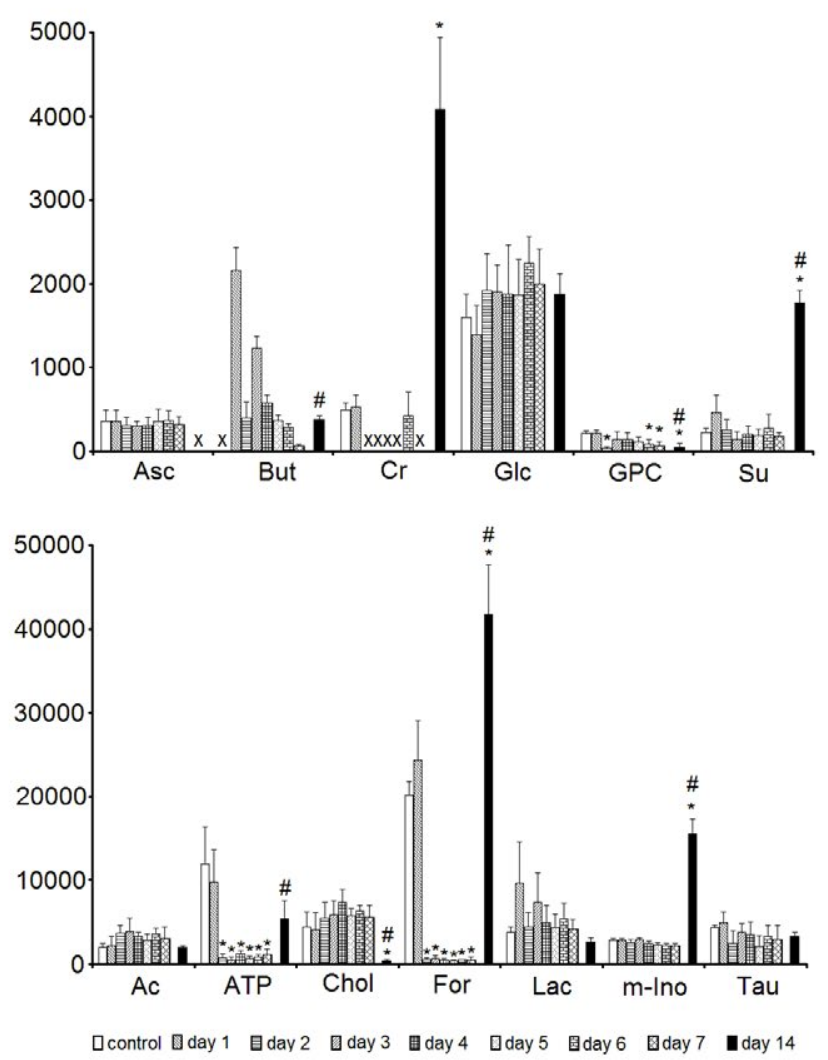

Figure 4. The effect of time of storage on the contents of assigned low molecular weight non-amino acid metabolites in corneas. Data are means \pm SEM (NMR peak integrals $/ \mathrm{mg}$ tissue). ${ }^{*}$-p $<0.05 v s$. control; \#-p $<0.05$, tissue preserved for 14 days $v s$. the ones preserved for 7 days; $X$ - no detectable metabolite peak in the respective NMR spectra. For metabolite labels see legend for Figure 1.

lachrymal fluid being the mixture of several compounds or secretions from glands located in the tissues circumventing the eye [16,17]. In this regard the hypothermic storage system based on a very basic commercially available medium such as Eusol-C [18], does not even approximate the in vivo conditions.

In fact, the hypothermic storage was, and it is, recognized as a method of preservation of the in vivo properties of human corneas in a mechanism based on the influence of low temperature $\left(2-8^{\circ} \mathrm{C}\right)$ on metabolic activities in the tissue cells: it was supposed to reduce cellular demand for metabolic energy $[11,15,19]$. According to this assumption the level of ATP in the tissue should remain the same or, considering lower energy demand, even increase with the storage time. To our surprise the results of this study has not confirmed this view as the levels of ATP in corneas preserved for 2-7 days were significantly lower than in the control samples (Figure 4). We have no clear evidencebased explanation for such observations. Maybe, and it is speculative at the moment, ATP as an energy source was displaced and utilized to maintaining the primary, at least, enzymatic systems in cells. The enzymatic systems aiming to protect the cells/tissue from damage resulting from their exposure to artificial and potentially stressful environment. This consumption of ATP was not counterbalanced by its production, possibly due to the low temperature of the storage medium. Interestingly, a similar pattern of energy changes was earlier observed by Redbrake et al. [20]. In that report the storage time was also correlated with an increase of a number of the damaged endothelial cells in the tissue, which was not observed in our study.

The very similar to ATP kinetics of the concentration changes revealed formate (Figure 4). This biochemical compound serves among others as a free radicals scavenger protecting cells from the oxidative damage [21]. Its deprivation in the tissue during the first week of the storage could suggest an accelerated utilization of this compound in response to the exposure of cornea cells to free radical species. However, taurine and ascorbate, two powerful antioxidants, remained unchanged during the storage (Figure 4). Then, the influence of free radicals was moderate and other factors could induce intracellular imbalance that caused deprivation of ATP in the first week of the storage (Figure 4). One of them could be the biochemical composition of the circumventing storage medium deficient in some biochemicals/ metabolites that were accessible in vivo [18].

The principal component analysis revealed that both, ATP and formate were identified (with minor influence of glycine) as the main biochemicals/components of $\mathrm{PC} 1$ responsible for the distant grouping of corneas preserved for 1 week from the control samples and 2-week samples (Figure 2). Furthermore, though the main PC2 components were identified, namely butyrate and lactate, no further separation of the samples in the score plot was observed (Figure 2). Then, PCA revealed the biochemical similarity between the corneas preserved in Eusol-C for one week, while the corneas preserved for 2 weeks showed a similar biochemical pattern to the initial values of control corneas (Figure 2). Similar, but not identical: the detailed statistical analysis revealed the biochemical differences within the group of corneas preserved for one week, as well as between the ones stored for 2 weeks and the control samples (Figures 3 and 4). Usually the levels of the most biochemical compounds remained stable or were decreasing during the first week of storage (see: ATP, formate, glycerophosphocholine, methionine; Figure 4) even down to undetectable values (see: creatine, glutamate, phenylalanine; Figures 3 and 4). The molecular mechanisms associated with these changes remained unclear as well as the influence of these differences on functioning of cells in the Eusol-C-based cold storage system. Some of these biochemical compounds showed the tendency of returning to the initial concentration values of the control corneas after two weeks of the preservation (see: ATP, methionine, phenylalanine; Figures 3 and 4). In addition, some of them showed significantly higher concentration values after two weeks of the storage, even in comparison to the control (see: creatine and formate; Figure 4) or the level of the biochemical compound was elevated even if no concentration changes were revealed within the first week of the storage (see: myo-inositol and succinate; Figure 4). On the other hand the levels of a few compounds were decreased after 2 weeks of the storage, even if no strongly marked changes were observed in the first week of the storage (see: alanine, glycine, ascorbate, glycerophosphocholine and choline; Figures 3 and 4). The above observations lead to a fundamental question: what is more advantageous for the cornea graft from the biochemical point of view - one week or two weeks of the storage? The question becomes even more crucial as the recent reports have highlighted the feasibility of transferring the donor tissues from cold storage to warm organ culture to increase the lifespan of donor corneas [13,14], but the issue is to make it in the best possible time-point from the metabolic point of view.

The answer is not so clear, as the changes referred to a broad spectrum of metabolites have usually nothing in common between themselves in the metabolic pathways. In addition some of them were the components of Eusol-C, used in this study [18]. In this regard, one observation seems of great importance: the levels of amino acids 
after the two-weeks storage were the same or significantly decreased in comparison to the control. Lowering levels of free amino acids could indicate their degradation in the tissue that was not equalized by their influx from the medium. On the other hand, it could also be the effect of the synthesis of proteins in the corneal cells with the rate exceeding the uptake of amino acids from Eusol-C. This process would indicate the restoring of the cellular and molecular properties of the corneas. The additional argument for this thesis seems to be the levels of glycerophosphocholine and choline assigned in two-weeks corneas (Figure 4). Both compounds are the components of the cell membranes $[22,23]$; their decrease would indicate their intense incorporation to the membranes and then, the consolidation of the cellular compartments of the tissue. The easier, as one of the principal metabolites in the cells, glucose, remained unchanged during the storage process (Figure 4). The latter probably resulted partially from its supplementation from the preservation medium that approximated the rate of its tissue consumption.

\section{Conclusions}

This study confirmed the previous reports $[1,2,5]$ that lowering the temperature to as low as $+4^{\circ} \mathrm{C}$ in the storage medium did not protect the cornea cells from metabolic changes. It was also shown that after two weeks of hypothermic Eusol-C storage the corneas seemed to be in a much better metabolic condition than the ones preserved for less than 7 days. It is not clear whether using another medium or storage type could affect these biochemical differences and how progressive or regressive they would become after the transplantation. A further research should explore this issue. Last but not least, the present results should open the discussion on the optimal time-windows for: (i) performing the cornea transplantation procedure or (ii) transferring the donor tissue from cold storage to organ culture to increase the lifespan of corneas.

\section{Acknowledgement}

The study was supported by the Research Council of Norway grant No. 170768, Mobility Stipend 2009 and Inger Holms Minnefond.

\section{References}

1. Kryczka T, Szaflik JP, Szaflik J, Midelfart A (2013) Influence of donor age, postmortem time and cold storage on metabolic profile of human cornea. Acta Ophthalmol 91: 83-7. [Crossref]

2. Kryczka T, Chrapusta SJ, Szaflik JP, Szaflik J, Midelfart A (2014) Impact of donor health on corneal biochemistry-an unexpected caveat from a pilot study. Ann Transplant 19: 129-37. [Crossref]

3. EEBA Technical Guidelines Special Interest Group (2005) Technical guidelines for ocular tissue.

4. The Eye Bank Association of America. Medical Standards (2003) Revision: D1.000 Donor Screening.
5. Kryczka T (2015) Cellular stress after transferring human cornea from in vivo to in vitro milieu - a metabolomic approach. Integr Mol 2: 234-237.

6. Kryczka T, Ehlers N, Nielsen K, Midelfart A (2012) Impact of organ culturing on metabolic profile of human corneas: preliminary results. Acta Ophthalmol 90: 761-7. [Crossref]

7. Pont T, Gràcia RM, Valdés C, Nieto C, Rodellar L, et al. (2003) Theoretic rates of potential tissue donation in a university hospital. Transplant Proc 35: 1640-1. [Crossref]

8. Tandon R, Verma K, Vanathi M, Pandey RM, Vajpayee RB (2004) Factors affecting eye donation from postmortem cases in a tertiary care hospital. Cornea 23: 597-601. [Crossref]

9. Gaum L, Reynolds I, Jones MN, Clarkson AJ, Gillan HL, et al. (2012) Tissue and corneal donation and transplantation in the UK. Br J Anaesth 108: i43-7.

10. Sharp C, Randhawa G (2014) Organ donation as an 'altruistic gift': Incentives and reciprocity in deceased organ donation from a UK Polish migrant perspective. Ann Transplant 19: 23-31. [Crossref]

11. Armitage WJ (2011) Preservation of Human Cornea. Transfus Med Hemother 38: 143 147. [Crossref]

12. Patel SV, Diehl NN, Hodge DO, Bourne WM (2010) Donor risk factors for graft failure in a 20-year study of penetrating keratoplasty. Arch Ophthalmol 128: 418-25. [Crossref]

13. Camposampiero D, Tiso R, Zanetti E, Ruzza A, Bruni A et al. (2003) Improvement of human corneal endothelium in culture after prolonged hypothermic storage. Eur $J$ Ophthalmol 13: 745-51. [Crossref]

14. Haug K, Azqueta A, Johnsen-Soriano S, Shahdadfar A, Drolsum LK et al. (2013) Donor cornea transfer from Optisol GS to organ culture storage: a two-step procedure to increase donor tissue lifespan. Acta Ophthalmol 91: 219-25. [Crossref]

15. Jeng BH (2006) Preserving the cornea: corneal storage media. Curr Opin Ophthalmol 17: 332-7. [Crossref]

16. Shoham A, Hadziahmetovic M, Dunaief JL, Mydlarski MB, Schipper HM (2008) Oxidative stress in diseases of the human cornea. Free Radic Biol Med 45: 1047-55. [Crossref]

17. Mantelli F, Argüeso P (2008) Functions of ocular surface mucins in health and disease. Curr Opin Allergy Clin Immunol 8: 477-83. [Crossref]

18. Alchimia srl. Media for corneal storage at +4 C. Eusol-C.

19. Pels E, Beele H, Claerhout I (2008) Eye bank issues: II. Preservation techniques: warm versus cold storage. Int Ophthalmol 28: 155-63. [Crossref]

20. Redbrake C, Becker J, Salla S, Stollenwerk R, Reim M (1994) The influence of the cause of death and age on human corneal metabolism. Invest Ophthalmol Vis Sci 35: 3553-6. [Crossref]

21. Shiraishi T, Fukusaki E, Miyake C, Yokota A, Kobayashi A (2000) Formate protects photosynthetic machinery from photoinhibition. J Biosci Bioeng 89: 564-568. [Crossref]

22. Zeisel SH, da Costa KA (2009) Choline: an essential nutrient for public health. Nutr Rev 67: 615-623. [Crossref]

23. Yen CL, Mar MH, Zeisel SH (1999) Choline deficiency-induced apoptosis in PC12 cells is associated with diminished membrane phosphatidylcholine and sphingomyelin, accumulation of ceramide and diacylglycerol, and activation of a caspase. FASEB $J 13$ : 135-142. [Crossref]

Copyright: (C2015 Sugitachi A. This is an open-access article distributed under the terms of the Creative Commons Attribution License, which permits unrestricted use, distribution, and reproduction in any medium, provided the original author and source are credited. 